\title{
A REGULATOR FOR CURVES VIA THE HEISENBERG GROUP
}

\author{
BY DINAKAR RAMAKRISHNAN
}

0. In this note we present a variation on a construction of P. Deligne [4] of the regulator map for $K_{2}$ of algebraic curves. This map, which may be viewed as an analog of the classical regulator map for the group of units in an algebraic number field, was first found for elliptic curves with complex multiplication by Spencer Bloch $[2,3]$. Deligne's method involves associating, to every pair of invertible holomorphic functions $f, g$ on a Riemann surface $X$, a holomorphic line bundle with connection on $X$, satisfying symbol properties. The new aspect of our construction is the use of a 'universal' line bundle with connection on $\mathbf{C}^{*} \times$ $C^{*}$ coming from a certain quotient $M$ of the complex three-dimensional Heisenberg group $H$. We were led naturally to $M$ while considering the transformation properties of the composite of dilogarithm with the elliptic modular function $\lambda$. Then Bloch suggested using it to define the regulator. The canonical way of defining the connection on $M$ evolved in conversation with him.

The main interest in this is the conjectural relationship to special values of zeta functions of curves over $\mathbf{Q}$. Some progress along these lines has been made by Bloch [2] in the case of elliptic curves, and by A. Beilinson [1] in the case of modular curves. A detailed exposition of these matters, starting from the method of this note and with some additions and machine calculations, will at some future date be published jointly with Bloch.

1. Let $V=\mathrm{C}^{2}, U=(2 \pi i \mathrm{Z})^{2} \subset V, B$ : a bilinear form on $V$ such that $B(U \times U) \subset(2 \pi i)^{2} \mathrm{Z}, e[y]=\exp \left[(2 \pi i)^{-1} y\right]$, and $H_{B}=V \times \mathrm{C}^{*}$ with multiplication $(v ; z) *_{B}\left(v^{\prime} ; z^{\prime}\right)=\left(v+v^{\prime} ; z z^{\prime} e\left[B\left(v, v^{\prime}\right)\right]\right)$. The left action of $U$ on $H_{B}$, given by $(u,(v ; z)) \rightarrow(u+v ; z e[B(u, v)])$, yields the identity $e\left[B\left(u+u^{\prime}, v\right)\right]=$ $e\left[B\left(u, u^{\prime}+v\right)\right] e\left[B\left(u^{\prime}, v\right)\right] \in \mathrm{C}^{*}$. Thus $u \rightarrow e[B(u,-)]$ is a 1-cocycle for $U$ with coefficients in $H^{0}\left(V, O_{V}^{*}\right)$ determining the class of the line bundle associated with the principal $\mathbf{C}^{*}$-bundle $M_{B} \stackrel{\text { def }}{=} U \backslash H_{B} \stackrel{\phi}{\rightarrow} \mathbf{C}^{*} \times \mathbf{C}^{*} \simeq U V$, where $\phi\left(\left(v_{1}, v_{2}\right) ; z\right)$ $=\left(e^{-v_{1}}, e^{v_{2}}\right)$ for all $\left(v_{1} v_{2}\right)$ in $V$ and $z$ in $C^{*}$. Set $B_{0}$ : the form with matrix $\left(\begin{array}{l}01 \\ 00\end{array}\right)$, and write $H=H_{B_{0}}, M=M_{B_{0}}$, etc. 
THEOREM. There exists a natural, holomorphic, nonintegrable connection $\nabla$ on $(M, \phi)$ such that, for every Riemann surface $X$ and invertible holomorphic functions $f, g$ on $X$, the pull-back $r(f, g) \stackrel{\text { def }}{=}(f, g) *(M, \phi ; \nabla)$, where $(f, g)$ denotes the map $X \rightarrow \mathrm{C}^{*} \times \mathrm{C}^{*}, x \rightarrow(f(x), g(x))$, defines a holomorphic line bundle on $X$ with integrable connection, satisfying

(a) $r\left(f, g g^{\prime}\right)=r(f, g) \cdot r\left(f, g^{\prime}\right)$,

(b) $r(f, g)=r(g, f)^{-1}$,

and

(c) $r(f, 1-f)=1$.

Proof. (I) Construction of $\nabla$ on $M$. The space of holomorphic sections of the trivial line bundle on $V$ coming from the abelianization map $\widetilde{\phi}: H_{B} \rightarrow V$, $(v ; z) \rightarrow v$, is as usual identified with the right $H_{B}$-module $\widetilde{L}_{B}=\left\{\sigma: H_{B} \rightarrow \mathrm{Cl} \sigma:\right.$ holomorphic and $\sigma(v ; z)=z \sigma(v ; 1)\}$. It is stable under Lie $H_{B}$. Identifying Lie $V$ with $\mathbf{C} \partial / \partial v_{1} \oplus \mathbf{C} \partial / \partial v_{2}$, we have the exact diagram

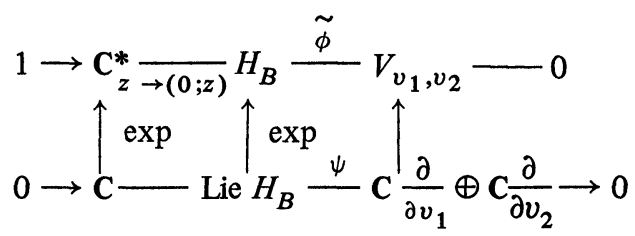

If $(v ; \xi) \in \operatorname{Lie} H_{B}$, then $\exp (v ; \xi)=(v ; e[\xi+1 / 2 B(v, v)])$. Clearly $\psi$ admits a natural section $s$ given by $\partial / \partial v_{1} \rightarrow\left(e_{1} ; 0\right)$ and $\partial / \partial v_{2} \rightarrow\left(e_{2} ; 0\right)$, where $e_{1}=$ $(1,0)$ and $e_{2}=(0,1)$ in $V$. Thus Lie $V$ acts on $\widetilde{L}_{B}$ by $(T, \underset{\sim}{\sigma} \rightarrow d \rho(s(T)) \sigma$. (Here $\rho$ denotes right translation.) This gives a connection $\widetilde{\nabla}_{B}$ on $\left(H_{B}, \underset{\widetilde{\phi}}{\widetilde{T}}\right.$ ). A simple computation yields that, for $\sigma$ in $\widetilde{L}_{B}, \widetilde{\nabla}_{B}(\sigma)=d \sigma+\widetilde{\nabla}_{B}(1) \sigma$ and $\widetilde{\nabla}_{B}(1)=$ $(2 \pi i)^{-1}\left(B\left(-, e_{1}\right) d v_{1}+B\left(-, e_{2}\right) d v_{2}\right)$.

Properties. (i) $\left(H_{B}, \widetilde{\nabla}_{B}\right) \otimes\left(H_{B^{\prime}}, \widetilde{\nabla}_{B^{\prime}}\right) \simeq\left(H_{B+B^{\prime}} ; \widetilde{\nabla}_{B+B^{\prime}}\right)$,

(ii) $B=C+{ }^{t} C, C \in M_{2}(\mathrm{Z}) \Rightarrow \widetilde{\nabla}_{B}(1)=d h$ for some $h \in \widetilde{L}_{B}$,

(iii) $\widetilde{\nabla}(1) \stackrel{\text { def }}{=} \widetilde{\nabla}_{B_{0}}(1)=(2 \pi i)^{-1} v_{1} d v_{2}$.

(iv) Every $\tau$ in $G L(2, \mathrm{Z})$ gives rise to an isomorphism $[\tau]: H_{\tau B} t_{\tau} \simeq H_{B}$ by $(v ; z) \rightarrow(v \tau ; z)$. Denoting the Weyl element $\left(\begin{array}{c}01 \\ 10\end{array}\right)$ by $w$, we have $(H, \widetilde{\nabla}) \otimes$ $[w] *(H, \widetilde{\nabla})=\left(H_{w}, \widetilde{\nabla}_{w}\right)$. (Here we use $w B_{0}^{t} w=w-B_{0}$.)

Next we identify the sections of $\left(M_{B}, \phi\right)$ with $L_{B}=\left\{\sigma \in \tilde{L}_{B} \mid \sigma\right.$ : invariant under the left action of $U\}$, the space of "degenerate theta functions". This is stable under $d \rho\left(\operatorname{Lie} H_{B}\right)$. And the differential of the map $V \stackrel{\eta}{\rightarrow} \mathbf{C}^{*} \times \mathbf{C}^{*}$, $\left(v_{1}, v_{2}\right) \rightarrow\left(e^{-v_{1}}, e^{v_{2}}\right)$, identifies the respective tangent spaces at the origin. Thus the left invariant derivations on $\mathbf{C}^{*} \times \mathrm{C}^{*}$ act on $L_{B}$ via the composite

$$
\operatorname{Lie}\left(\mathrm{C}^{*} \times \mathrm{C}^{*}\right) \stackrel{d \eta^{-1}}{\longrightarrow} \operatorname{Lie}(V) \stackrel{s}{\longrightarrow} \operatorname{Lie}\left(H_{B}\right) \stackrel{d \rho}{\longrightarrow} \text { End } L_{B} .
$$

This gives the desired connection $\nabla_{B}$ on $\left(M_{B}, \phi\right)$. 
LOCAL DESCRIPTION. Let $\left\{O_{j}\right\}$ be an open cover of $\mathbf{C}^{*}$ with single-valued branches $\log _{j}$ of logarithms defined on the $O_{j}$ 's. Then,

$$
\nabla_{B}\left(1_{O_{j} \times O_{k}}\right)=(2 \pi i)^{-1} \cdot\left[\begin{array}{c}
B\left(\left(\log _{j} y_{1}, \log _{k} y_{2}\right), e_{1}\right) d \log _{j} y_{1} \\
+B\left(\left(\log _{j} y_{1}, \log _{k} y_{2}\right), e_{2}\right) d \log _{k} y_{2}
\end{array}\right] \text {. }
$$

In particular, $\nabla\left(1_{O_{j} \times O_{k}}\right)=(2 \pi i)^{-1} \log _{j} y_{1} d \log _{k} y_{2}$.

The properties (i)-(iv) of $\widetilde{\nabla}_{B}$ pass to the quotient and remain valid for $\nabla_{B}$. The nonintegrability of $\nabla$ comes from $s$ not being a Lie algebra section. If we change the section $s$, then $\nabla$ gets modified by an integrable connection.

(II) Bilinearity, skew-symmetry of $r$. It is clear that $r\left(f, g g^{\dagger}\right)=r(f, g) \cdot$ $r\left(f, g^{\prime}\right)$. And the commutativity of the diagram

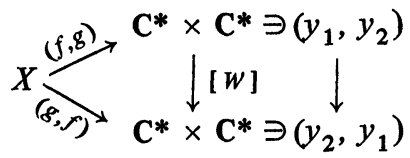

yields that $r(g, f)=(f, g) *([w] *(M, \nabla))$.

Then, by using property (iv), we get $r(f, g) \cdot r(g, f)=(f, g)^{*}\left(M_{w}, \nabla_{w}\right)$. By property (ii), $\nabla_{w}$ is trivial since $w$ equals $B_{0}+{ }^{t} B_{0}$.

(III) Steinberg relation. It is enough to show that $r(1-\mathrm{id}$, id $)=1$ on $\mathbf{P}^{1}$ $-\{0,1, \infty\}$.

Define recursively the higher logarithms $\ln _{k}$ by $\ln _{k}(x)=\int_{0}^{x} \ln _{k-1}(t) d t / t$, with $\ln _{0}(x)=x /(1-x)$. Then $\ln _{1}(x)=\log (1 /(1-x))$, and $\ln _{2}$ satisfies the 3 rd order, homogeneous, algebraic differential equation

$$
\frac{d}{d x}(1-x) \frac{d}{d x} x \frac{d}{d x} u=0 .
$$

It has regular singular points at $\{0,1, \infty\}$. The 'principal branch' of $\ln _{2}$ in the unit disk is given by $\Sigma_{n \geqslant 1} x^{n} / n^{2}$. Let

$$
N=\left\{\left(\begin{array}{ccc}
1 & x & z \\
0 & 1 & y \\
0 & 0 & 1
\end{array}\right) \mid x, y, z \in \mathrm{C}\right\}
$$

and

$$
\Gamma=\left\{\left(\begin{array}{ccc}
1 & a & c \\
0 & 1 & b \\
0 & 0 & 1
\end{array}\right) \mid \begin{array}{c}
a, b \in 2 \pi i \mathbf{Z} \\
c \in(2 \pi i)^{2} Z
\end{array}\right\}
$$


Proposition. (i) The map $\mathbf{P}^{1}-\{0,1, \infty\} \stackrel{\beta}{\rightarrow} N$, given by

$$
x \rightarrow\left(\begin{array}{ccc}
1 & \ln _{1} x & \ln _{2} x \\
0 & 1 & \log x \\
0 & 0 & 1
\end{array}\right)
$$

is well defined modulo $\Gamma$.

(ii) The diagram

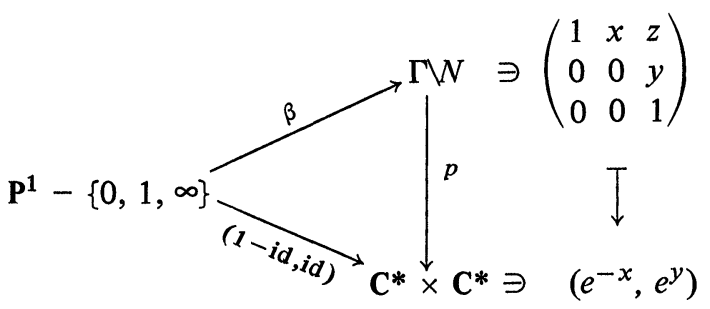

is commutative.

We are done modulo this proposition. Let $\alpha$ denote the canonical analytic isomorphism

$$
\Gamma N \cong U H, \quad\left(\begin{array}{ccc}
1 & x & z \\
0 & 1 & y \\
0 & 0 & 1
\end{array}\right) \rightarrow((x, y) ; e[z])
$$

Then the proposition implies that $\phi(\alpha(\beta(x)))=(1-x, x)$, for every $x$ in $\mathbf{P}^{\mathbf{1}}-$ $\{0,1, \infty\}$. Let $O$ be an open set in $\mathbf{P}^{1}-\{0,1, \infty\}$ where a single-valued branch of $\ln _{1}$ is defined. Then the connection equation on $O$ is

$$
\nabla \sigma(x)=\frac{1}{2 \pi i} \log (1-x) d \log x \cdot \sigma+d \sigma .
$$

So

$$
\sigma \text { is horizontal } \Longleftrightarrow \sigma=\exp \left(\frac{1}{2 \pi i} \int_{0}^{x} \ln _{1}(t) \frac{d t}{t}\right) .
$$

Clearly $\alpha \circ \beta$ yields such a (horizontal) section.

Now we will prove the proposition. The solution space to $(*)$ is spanned by $\left\{\ln _{2}, \log , 1\right\}$. It is straightforward to check that the monodromy around 0 (resp. 1) is given by

$$
\left(\begin{array}{c}
\ln _{2} \\
\log
\end{array}\right) \rightarrow\left(\begin{array}{l}
\ln _{2} \\
\log +2 \pi i
\end{array}\right)\left(\operatorname{resp} \cdot\left(\begin{array}{l}
\ln _{2} \\
\log \\
1
\end{array}\right) \rightarrow\left(\begin{array}{c}
\ln _{2}+2 \pi i \log \\
\log
\end{array}\right)\right)
$$


Thus we get a unipotent 3-dimensional representation of $\pi_{1}\left(\mathbf{P}^{1}-\{0,1, \infty\}\right)$, whose image is precisely $\Gamma$. This gives a map of $\mathbf{P}^{1}-\{0,1, \infty\}$ into $\Gamma \mathbf{C}^{2}$, by $x \rightarrow\left(\begin{array}{l}\ln _{2} x \\ \log x\end{array}\right)$. (This is unsatisfactory because $\Gamma$ does not act freely on $\mathbf{C}^{2}$.)

Considering the differential equation of $\ln _{1}$, we get the corresponding monodromy around 0 (resp. 1) to be

$$
\left(\begin{array}{c}
\ln _{1} \\
1
\end{array}\right) \rightarrow\left(\begin{array}{c}
\ln _{1} \\
1
\end{array}\right) \quad\left(\operatorname{resp} \cdot\left(\begin{array}{c}
\ln _{1} \\
1
\end{array}\right) \rightarrow\left(\begin{array}{c}
\ln _{1}+2 \pi i \\
1
\end{array}\right)\right) .
$$

This makes it clear that the map $x \rightarrow \beta(x)$ is well defined modulo $\Gamma$.

The commutativity of the diagram in part (ii) of the proposition is now immediate. Q.E.D.

Using Matsumoto's presentation of $K_{2}$ of a field in terms of symbols, we immediately obtain the following.

CoRollary. Let $X$ be a smooth, projective algebraic curve over $k \subset \mathrm{C}$. Then $r$ induces a homomorphism

$$
K_{2}(k(X)) \rightarrow \lim _{\substack{S \subset X_{\mathrm{C}} \\ \text { finite }}} H^{1}\left(X_{\mathrm{C}}-S, \mathrm{C}^{*}\right)
$$

For the extension of this to the global regulator map from $K_{2}(X)$ into $H^{1}\left(X_{C}, C^{*}\right)$ and relations to the Kac-Moody Lie algebras, see Bloch's Dilogarithm and extensions of Lie algebras (to appear).

\section{BIBLIOGRAPHY}

1. A. A. Beilinson, Higher regulators and values of L-functions of curves, J. Functional Anal. Appl. 14 (1980), 116-118.

2. S. Bloch, Higher regulators, algebraic $K$-theory and zeta functions of elliptic curves, preprint.

3. - Lectures on algebraic cycles, Duke University Mathematics Series. IV

4. P. Deligne, Le symbole modéré, unpublished.

DEPARTMENT OF MATHEMATICS, UNIVERSITY OF CHICAGO, CHICAGO, ILLINOIS 60637 
